\title{
Impacto dos Aspectos Sociodemográficos e Clínicos na Qualidade de Vida de Portadores de HTLV-I com HAM/TSP
}

\author{
Luana Mocellin Delazeri \\ Escola Bahiana de Medicina e Saúde Pública \\ Luana Mocellin Delazeri \\ Luana Rodrigues Santos \\ Escola Bahiana de Medicina e Saúde Pública \\ (EBMSP) \& Grupo de Pesquisa em Dinâmica do \\ Sistema Neuromusculoesquelético / EBMSP \\ Escola Bahiana de Medicina e Saúde Pública \\ Selena Márcia Dubois Mendes \\ (EBMSP) \& Grupo de Pesquisa em Dinâmica do \\ Sistema Neuromusculoesquelético / EBMSP \\ Abrahão Fontes Baptista \\ Grupo de Pesquisa em Dinâmica do Sistema \\ Neuromusculoesquelético / EBMSP \\ Escola Bahiana de Medicina e Saúde Pública \\ Kátia Nunes Sá \\ EBMSP) \& Grupo de Pesquisa em Dinâmica do \\ Sistema Neuromusculoesquelético / EBMSP \\ Bernado Galvão-Castro \\ Escola Bahiana de Medicina e Saúde Pública \\ (EBMSP) \\ Tipo de Análise do Manuscrito \\ Triple Blind Peer Review \\ Recebido em Dez/2011 Aprovado em Dez 2011
}

\section{RESUMO}

Introdução: 0 vírus linfotrópico de células T Humanas - HTLV-1, retrovírus humano, pode provocar, dentre outras, uma doença neurológica: a paraparesia espástica tropical / mielopatia associada ao HTLV-1 (HAM/TSP). É uma doença progressiva e incapacitante podendo gerar distúrbios da marcha, fraqueza muscular e enrijecimento dos membros inferiores, além de comprometimento do equilíbrio, o que impacta na qualidade de vida das pessoas infectadas. Objetivo: Verificar a associação dos domínios da qualidade de vida com aspectos sociodemográficos e clínicos em portadores de HTLV-I com HAM/TSP. Métodos: Estudo observacional de corte transversal em amostra de 115 portadores de HTLV-1 com HAM/TSP de um centro de referência. Os dados foram verificados através do questionário SF36 e de uma ficha de dados sociodemográficos e clínicos. Utilizou-se o teste Qui-Quadrado para testar o nível de significância entre as variáveis: idade categorizada, cor da pele, classe social, estado civil, escolaridade e diagnóstico de HAM/TSP categorizados, os quais foram relacionados com os domínios de Qualidade de Vida do questionário SF-36. 0 teste t-student foi utilizado na pesquisa para testar a relação existente entre as variáveis: sexo e atividade física e fisioterapia associado com os domínios da Qualidade de Vida. Resulta dos: Verificou-se que $69,6 \%$ dos participantes eram do sexo feminino, $72,2 \%$ tinham idade entre 35 a 64 anos, $59,1 \%$ pertenciam à classe social D e $29,6 \%$ tinham escolaridade baixa. Quando comparados os domínios da qualidade de vida com o diagnóstico de HAM/TSP, verificou-se maiores comprometimentos em capacidade funcional $(\mathrm{p}=0,001)$ e aspecto físico $(\mathrm{p}=0,002)$. Pertencer ao gênero feminino e à classe social baixa geraram maior perda da qualidade de vida. Conclusão: Os domínios da QV mais comprometidos nos portadores de HTLV-1 com HAM/TSP definido foram capacidade funcional e aspecto físico. As pessoas mais comprometidas foram adultos do sexo feminino pertencentes à classe social D.

Palavras Chave: Virus Linfotropico, HAM-TSP, HTLV-1 


\section{Impact of Socio-demographic and Clinical Aspects in Quality of Life of Patients with HTLV-I HAM / TSP}

\section{ABSTRACT}

Introduction: The T-cell lymphotropic virus Humanities - HTLV-1, human retrovirus, can cause, among others, a neurological disorder, tropical spastic paraparesis / myelopathy associated with HTLV-1 (HAM / TSP). It is a progressive and debilitating and may cause gait disturbances, muscle weakness and stiffness of the lower limbs, and impaired balance, which impacts the quality of life of people infected. Objective: To investigate the association domains of quality of life with sociodemographic and clinical aspects in patients with HTLV-I HAM / TSP. Methods: Observational study of cross-sectional sample of 115 patients with HTLV-1 with HAM / TSP in a reference center. These data were verified using the SF-36 and form a demographic and clinical data. We used the chi-square test to test the level of significance between the variables: age categorized, skin color, social class, marital status, education and diagnosis of HAM / TSP categorized, which were related to the areas of Quality of Life of the SF-36. The Student's t test was used in research to test the relationship between the variables: sex, physical activity and physical therapy associated with the domains of quality of life. Results: It was found that $69.6 \%$ of participants were female, $72.2 \%$ were aged 35-64 years, $59.1 \%$ belonged to social class D and $29.6 \%$ had low education. When comparing the domains of quality of life with the diagnosis of HAM / TSP, there was greater impairment in functional capacity $(p=0.001)$ and physical appearance $(p=0.002)$. Belonging to the female gender and low social class have generated greater loss of quality of life. Conclusion: The most affected areas of QoL in patients with HTLV-1 with HAM / TSP were defined functional capacity and physical appearance. The people most affected were adult females belonging to the class D.

Keywords: lymphotropic virus, HAM-TSP, HTLV-1

\section{Impacto de los aspectos socio-demográficas y clínicas en la calidad de vida de los pacientes con HTLV-I HAM / TSP}

\section{RESUMEN}

Introducción: Las Humanidades linfotrópico de células T del virus - el HTLV-1, retrovirus humanos, puede causar, entre otros, un trastorno neurológico, paraparesia espástica tropical / mielopatía asociada con el HTLV-1 (HAM / TSP). Se trata de una progresiva y debilitante y puede causar alteraciones de la marcha, debilidad muscular y rigidez de las extremidades inferiores y alteración del equilibrio, lo cual afecta la calidad de vida de las personas infectadas. Objetivo: Investigar los dominios de la asociación de la calidad de vida de los aspectos sociodemográficos y clínicos en pacientes con HTLV-I HAM / TSP. Métodos: Estudio observacional de corte transversal muestra de 115 pacientes con HTLV-1 con HAM / TSP en un centro de referencia. Estos datos fueron verificados mediante el SF-36 y formar una base de datos demográficos y clínicos. Se utilizó la prueba de chi-cuadrado para probar el nivel de significación entre las variables: edad categorizadas, color de piel, clase social, estado civil, la educación y el diagnóstico de HAM / TSP categoría, que se relacionan con las áreas de Calidad de La vida de la SF-36. Prueba de la t de Student se utilizó en la investigación para probar la relación entre las variables: sexo, actividad física y la terapia física asociada a los dominios de la calidad de vida. Resultados: Se encontró que $69,6 \%$ de los participantes eran del sexo femenino, 72,2\% tenían entre 35-64 años, 59,1\% pertenecía a la clase social D y 29,6\% tenían bajo nivel de educación. Al comparar los ámbitos de la calidad de vida con el diagnóstico de HAM / TSP, hubo un mayor deterioro en la capacidad funcional $(p=0,001)$ y la apariencia física $(p=0,002)$. La pertenencia al género femenino y la clase social baja, han generado una mayor pérdida de calidad de vida. Conclusión: Las zonas más afectadas de la calidad de vida en pacientes con HTLV-1 con HAM / TSP se define la capacidad funcional y la apariencia física. Las personas más afectadas fueron las mujeres adultas que pertenecen a la clase D.

Palabras clave: Virus linfotrópico, HAM-TSP, HTLV-1 


\section{Impact des aspects socio-démographiques et cliniques dans la qualité de vie des patients atteints de HTLV-I HAM / TSP}

\section{RÉSUMÉ}

Introduction: Les sciences humaines T-virus lymphotrope - HTLV-1, un rétrovirus humain, peut causer, entre autres, un trouble neurologique, la paraparésie spastique tropicale / myélopathie associée à HTLV-1 (HAM / TSP). Il s'agit d'une progressive et débilitante et peut provoquer des troubles de la marche, une faiblesse musculaire et la raideur des membres inférieurs, et altération de l'équilibre, ce qui affecte la qualité de vie des personnes infectées. Objectif: étudier les domaines d'association de la qualité de la vie avec les aspects sociodémographiques et cliniques chez les patients atteints HTLV-I HAM / TSP. Méthodes: Etude observationnelle de l'échantillon transversal de 115 patients atteints de HTLV-1 avec HAM / TSP dans un centre de référence. Ces données ont été vérifiées à l'aide du questionnaire SF36 et forment un ensemble de données démographiques et cliniques. Nous avons utilisé le test du chi-carré pour tester le niveau de signification entre les variables: l'âge classés, couleur de la peau, la classe sociale, l'état matrimonial, l'éducation et le diagnostic de la HAM / TSP classés, qui ont été liés aux domaines de la qualité de Vie du SF-36. Le test t de Student a été utilisé dans la recherche pour tester la relation entre les variables: le sexe, l'activité physique et la physiothérapie associée à des domaines de la qualité de vie. Résultats: On a constaté que 69,6\% des participants étaient des femmes, 72,2\% étaient âgés de 35-64 ans, $59,1 \%$ appartenaient à la classe sociale D et 29,6\% avaient fait des études à faible. Lorsque l'on compare les domaines de la qualité de la vie avec le diagnostic de la HAM / TSP, il y avait une plus grande déficience de la capacité fonctionnelle $(\mathrm{p}=0,001)$ et l'apparence physique $(\mathrm{p}$ $=0,002$ ). Appartenant au genre féminin et basse classe sociale ont généré une plus grande perte de qualité de vie. Conclusion: Les zones les plus touchées de la qualité de vie chez les patients atteints du HTLV-1 avec HAM / TSP ont été définis de la capacité fonctionnelle et l'apparence physique. Les personnes les plus touchées étaient des femmes adultes appartenant à la classe $\mathrm{D}$.

Mots-clés: virus lymphotrope, HAM-TSP, HTLV-1

\section{Introdução}

Após vinte anos de identificação do HTLV-I, foram verificadas altas taxas endêmicas em diferentes áreas geográficas como o sudoeste do Japão, ilhas do Caribe (Jamaica e TrinidadTobago), a América do Sul, a África equatorial e Oceania (Papua Nova Guiné).1 No Brasil os primeiros relatos de infecção por HTLV-I aconteceram a partir de 1989. Estudos identificaram a presença do vírus, inicialmente, em 1,8\% de indivíduos na população geral2; em 1,35\% dos doadores de sangue 3 e 1,76\% da população em geral4. Estima-se que cerca de 40.000 pessoas nesta cidade são portadoras do vírus, sendo assim representada como maior prevalência no Brasil com incidência ligeiramente elevada em idade adulta e raça negra.4

Esse vírus pode ser adquirido através de transmissão horizontal (transfusão sangüínea, contato sexual, uso comum de seringas contaminadas) ou vertical, através do aleitamento materno.5,6 Quanto à fisiopatologia da paraparesia espástica tropical / mielopatia associada ao HTLV-1 (HAM/TSP) é causada pelo linfocitotrópico que interfere no funcionamento da imunidade celular. Os linfócitos T são ativados na paraparesia espástica tropical, atravessam a barreira hemato-encefálica, iniciando assim o processo inflamatório no SNC que resulta em lesão celular. 0 resultado é a desmielinização e degeneração axonal em níveis baixos da medula, provocando comprometimentos motores (fraqueza e espasticidade em membros inferiores); sensitivos (parestesias e dores neuropáticas), distúrbios esfincterianos vesicais e intestinais, além de disfunção erétil no homem. 5 
As principais patologias associadas são: leucemia / linfoma de células $\mathrm{T}$ do adulto (LLTA) e paraparesia espástica tropical / mielopatia associada ao HTLV-1 (HAM/TSP).1 A Organização Mundial de Saúde (OMS) propôs critérios clínicos para o diagnóstico de HAM/TSP, considerando idade, sexo, início da doença, principais manifestações neurológicas e sistêmicas, assim como diagnóstico laboratorial. Neurologistas brasileiros e de outros países propuseram um novo modelo complementar ao critério diagnóstico da OMS, que demonstra os níveis de desenvolvimento dos sintomas da doença classificados em: definido, provável e possível. 7

Sabe-se que a Qualidade de Vida (QV) recebe diferentes definições na literatura, incorporando distintos significados para pessoas, culturas, tempos e áreas de aplicação, tornando difícil a obtenção de uma única definição. 8 De acordo com a Organização Mundial da Saúde (OMS) QV é: "a percepção do indivíduo de sua posição na vida no contexto da cultura e sistema de valores nos quais ele vive e em relação aos seus objetivos, expectativas, padrões e preocupações.The WHOQOL Group em 1996 sugeriu que "essa definição deixa implícita a idéia de que o conceito de QV é subjetivo, multidimensional e inclui elementos de avaliação tanto positivos como negativos.9,10

A avaliação de qualidade de vida torna-se uma importante medida para assegurar uma adequada atenção às intervenções e às doenças que, mesmo não levando à morte iminente, causam grande desconforto ao paciente. A qualidade de vida ligada à saúde pode ser definida como o valor atribuído à vida, pelas deteriorações funcionais; as percepções e condições sociais que são induzidas pela doença, agravos, tratamentos e a organização política e econômica do sistema assistencial. 8

Desenvolvido no final dos anos 80, o questionário SF-36 - Medical Outcomes Study 36 Item Short-Form Health Survey, utilizado para verificar a QV, é um instrumento validado em várias circunstâncias para medir a qualidade de vida, cuja estrutura dimensional oferece boa representatividade da situação de saúde. Apresenta boa sensibilidade e pode ser aplicado em diversas situações, desde estabelecimentos de saúde até inquéritos populacionais. 11

Tendo em vista à alta prevalência do HTLV-I em Salvador, uma área endêmica, bem como o impacto na qualidade de vida desses pacientes, o objetivo desse estudo consiste na associação da qualidade de vida em portadores de HTLV-I com HAM/TSP entre aspectos demográficos, clínicos e classificação diagnóstica.

\section{Material \& Método}

Este estudo é caracterizado como transversal do tipo observacional, contendo uma amostra formada por 115 pacientes portadores de HTLV-1 que apresentam HAM/TSP do Centro de Referencia Integrado e Multidisciplinar de Indivíduos Infectados pelo HLTV (CHTLV) da Escola Bahiana de Medicina e Saúde Pública (EBMSP), Salvador-BA.

Inicialmente a amostra era constituída de 193 pacientes, foram exclusos 78 portadores que apresentavam déficits cognitivos ou não tinham diagnóstico confirmado para HAM/TSP. Este trabalho foi aprovado pelo Comitê de Ética em Pesquisa da Fundação Bahiana para Desenvolvimento das Ciências em Junho de 2008, referente ao protocolo 24/2008, respeitando os aspectos éticos, de confidencialidade e afirmando a ausência de riscos aos participantes, em concordância com o Conselho Nacional de Saúde (resolução 196/96).

Os dados sociodemográficos e clínicos foram verificados através de uma ficha, constituída de sexo, idade, estado civil, cor da pele auto definida, escolaridade, classe social, tempo de acompanhamento médico e realização de fisioterapia. Os itens escolaridade e classe socioeconômica foram avaliados pelo critério ABIPEME, sendo da classe social " $A$ " quando informaram 89 ou mais itens de conforto familiar, da classe social "B" de 59 a 88 itens, da classe social C de 35 a 58 itens, da classe social D de 20 a 34 itens e da classe social "E" de 0 a 19 itens. 
O questionário SF-36, utilizado para medir as variáveis QV, é composto por 11 questões e 36 itens que englobam oito componentes (domínios ou dimensões), que são: capacidade funcional, aspectos físicos, dor, estado geral da saúde, vitalidade, aspectos sociais itens, aspectos emocionais, saúde mental e uma questão comparativa sobre a percepção atual da saúde e há um ano, no qual, o indivíduo recebeu um escore em cada domínio, que variou de 0 a 100, sendo 0 o pior escore e 100 o melhor.

Utilizou-se o teste Qui-Quadrado para testar o nível de significância entre as variáveis: idade categorizada, cor da pele, classe social, estado civil, escolaridade, uso de dispositivo auxiliar e classificação do diagnóstico de HAM/TSP, os quais foram relacionados com os domínios de Qualidade de Vida do SF-36 categorizados em (0-25\%; 26-50\%; 51-75\%; 76$100 \%)$ de acordo com a distribuição percentual dos pacientes nos domínios do SF-36; lembrando que conforme aumenta a porcentagem, há melhor qualidade de vida e vice-versa. 0 teste t-student foi utilizado também nesta pesquisa para testar a relação existente entre as variáveis: sexo, atividade física e fisioterapia associado com os domínios da Qualidade de Vida do SF-36.

Os dados foram dispostos em tabelas ou gráficos e analisados pelo software Statistic Package for Social Science - SPSS (SPSS, Inc, Chicago, IL) versão 14.0 for Windows. As variáveis preditoras foram o perfil demográfico, clinico e o diagnóstico da HAM/TSP, e a de desfecho, foram os domínios da qualidade de vida do questionário SF-36.

\section{Resultados}

Verificou-se que $80(69,6 \%)$ pertenciam ao gênero feminino, quanto à faixa etária os indivíduos de meia idade (35 a 64 anos) representam 83 (72,2\%) da amostra. Relacionado ao estado civil a grande maioria são casados $47(41,2 \%)$, prevalecendo a cor da pele negra 53 $(46,1 \%)$. Em relação à atividade física $92(80,08 \%)$ não praticam exercícios regularmente, 81 $(70,4 \%)$ não realizam acompanhamento fisioterapêutico, enquanto que $74(64,3 \%)$ não fazem uso de quaisquer dispositivo auxiliar para marcha (Tabela 1).

Referente à classe social a amostra não possuía nenhum individuo da classe $\mathrm{A}$ e $\mathrm{B}$, sendo a maior prevalência concentrada na categoria D $68(59,1 \%)$, representado pelo critério ABIPEME como classe pobre, outra variável que foi apontado pelos níveis socioeconômicos baixos foi escolaridade, a qual houve maiores índices em Analfabeto/Primário incompleto 29 $(25,2 \%)$ e Primário completo/Ginásio incompleto 34 (29,6\%) (Tabela 1). 
Tabela 1

Características sociodemográficas e clínica da amostra de 115 portadores HTLV-1 com HAM-TSP do ADAB na cidade de Salvador-BA, 2011

\begin{tabular}{|c|c|}
\hline Variáveis & n (\%) \\
\hline \multicolumn{2}{|l|}{ Gênero } \\
\hline Feminino & $80(69,6)$ \\
\hline Masculino & $35(30,4)$ \\
\hline \multicolumn{2}{|l|}{ Idade } \\
\hline 20-34 anos & $17(14,8)$ \\
\hline 35 a 64 anos & $83(72,2)$ \\
\hline$>65$ anos & $15(13,0)$ \\
\hline \multicolumn{2}{|l|}{ Estado Civil } \\
\hline Solteiro & $40(35,1)$ \\
\hline Casado & $47(41,2)$ \\
\hline Viúvo & $12(10,5)$ \\
\hline \multicolumn{2}{|l|}{ Cor da pele auto-definida } \\
\hline Branco & $14(12,2)$ \\
\hline Negro & $53(46,1)$ \\
\hline Pardo & $46(40,0)$ \\
\hline \multicolumn{2}{|l|}{ Escolaridade* } \\
\hline Analfabeto/Primário incompleto & $29(25,2)$ \\
\hline Primário completo/Ginásio incompleto & $34(29,6)$ \\
\hline Ginásio completo/Colegial incompleto & $20(17,4)$ \\
\hline Colegial completo/Superior incompleto & $25(21,7)$ \\
\hline Superior Completo & $07(06,1)$ \\
\hline \multicolumn{2}{|l|}{ Classe Social* } \\
\hline Classe C & $23(20,0)$ \\
\hline Classe E & $24(20,9)$ \\
\hline \multicolumn{2}{|l|}{ Realiza atividade física } \\
\hline Sim & $23(20,0)$ \\
\hline Não & $92(80,0)$ \\
\hline \multicolumn{2}{|l|}{ Realização de fisioterapia } \\
\hline Não & $81(70,4)$ \\
\hline Sim & $34(29,6)$ \\
\hline \multicolumn{2}{|l|}{ Uso de dispositivo auxiliar } \\
\hline Nenhum & $74(64,3)$ \\
\hline Bengala & $09(07,8)$ \\
\hline Uma muleta & $09(07,8)$ \\
\hline Par de muletas & $07(06,1)$ \\
\hline Andador & $02(01,7)$ \\
\hline Cadeira de rodas & $14(12,2)$ \\
\hline
\end{tabular}

OBS: *Critério ABIPEME 
A qualidade de vida, avaliada segundo a média dos componentes do questionário SF-36 e os respectivos desvios padrão, mostra os seguintes resultados: capacidade funcional $(40.7+32.5)$; aspecto físico $(34.3+40.7)$; dor $(48,0+28,4)$; estado geral de saúde $(54.2+24.2)$; vitalidade $(48,9+28,4)$; aspecto social $(63.9+27.3)$; aspectos emocionais $(52.4+43.2)$ e saúde mental $(55.8+27.2)$, evidenciando o domínio aspecto físico como o mais comprometido nos pacientes com HTLV-I (FIGURA 1).

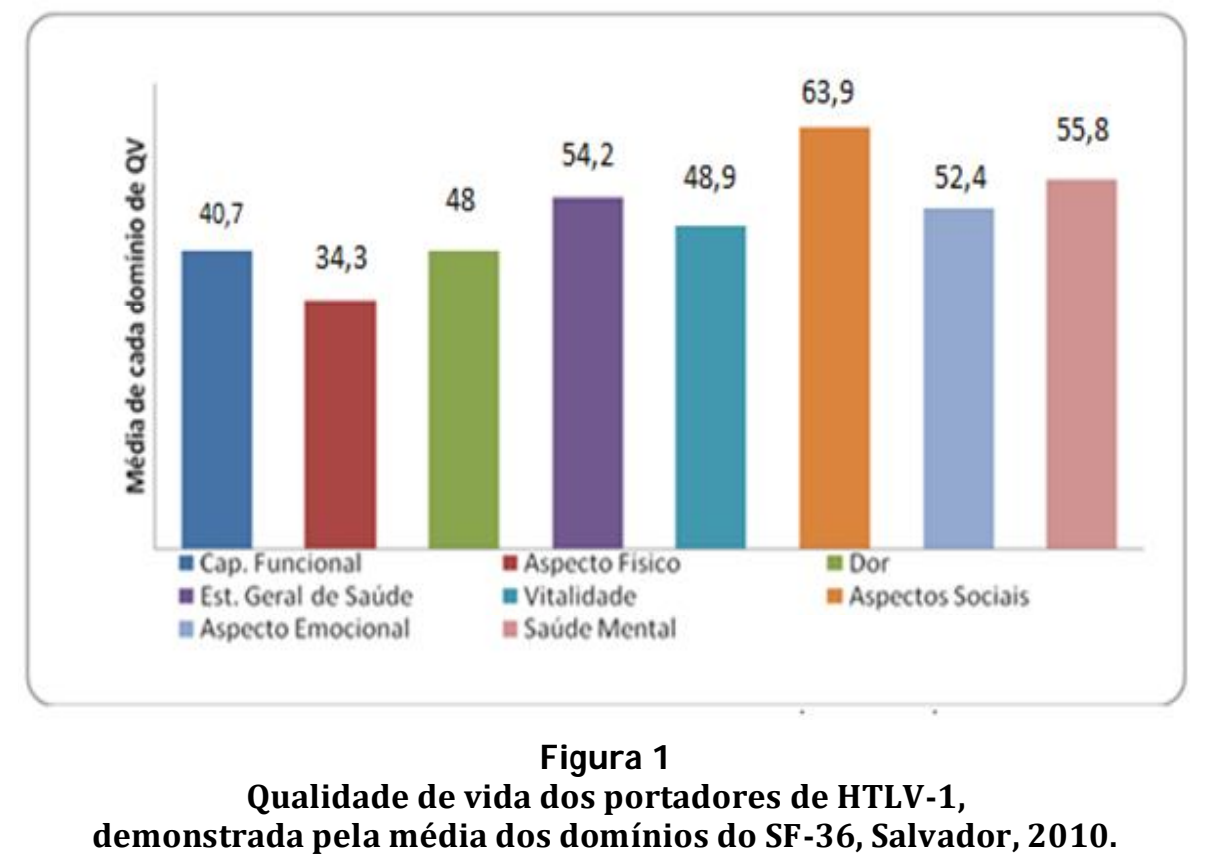

A variável sociodemográfica que apresentou correlação linear com algumas das dimensões da qualidade de vida foi o gênero. Essa variável correlacionou-se positivamente com as dimensões: Vitalidade e saúde mental, representados pelo $p<0,001$, outras dimensões que se relacionaram positivamente com o gênero foram aspectos sociais e emocionais com $\mathrm{p}=$ 0,017 e 0,012 respectivamente, relacionados com qualidade de vida (SF-36) (Tabela 2).

Em relação às variáveis clínicas, fazer ou não atendimento fisioterapêutico apresentou relação com os domínios do SF-36: capacidade funcional e aspectos físicos $\mathrm{p}<0,001$ e dor $\mathrm{p}=$ 0,005, o uso de dispositivo auxiliar também apresentou significância com os domínios da qualidade de vida, obtendo um maior nível de significância no domínio capacidade funcional p $=0,001$.

A análise da influência do gênero com os domínios vitalidade e saúde mental do SF-36, ressaltando que, conforme aumenta a porcentagem, há melhor qualidade de vida e vice-versa, o gênero feminino apresenta prevalência de porcentagens inferiores ao comparada com indivíduos do gênero masculino, mostrando as seguintes médias e os seus respectivos desvios padrão (Tabela 2). 
Tabela 2

Associação das variáveis sociodemográficas e clínicas com os domínios da Qualidade de Vida (SF-36) de 115 pacientes com HTLV-I (HAM/TSP), Salvador, 2010

\begin{tabular}{|c|c|c|c|c|c|c|c|c|}
\hline \multirow[b]{2}{*}{ Variáveis } & \multicolumn{8}{|c|}{ Domínios } \\
\hline & $\begin{array}{c}\text { Capacid } \\
\text { ade } \\
\text { funciona } \\
\text { | }\end{array}$ & $\begin{array}{l}\text { Aspecto } \\
\text { s Físicos }\end{array}$ & Dor & $\begin{array}{l}\text { Estado } \\
\text { Geral da } \\
\text { Saúde }\end{array}$ & $\begin{array}{c}\text { Vitalida } \\
\text { de }\end{array}$ & $\begin{array}{l}\text { Aspecto } \\
\text { s Sociais }\end{array}$ & $\begin{array}{l}\text { Aspecto } \\
\text { S } \\
\text { Emocion } \\
\text { ais }\end{array}$ & $\begin{array}{l}\text { Saúde } \\
\text { Mental }\end{array}$ \\
\hline *Gênero & 0,288 & 0,189 & 0,266 & 0,035 & $<0,001$ & 0,017 & 0,012 & $<0,001$ \\
\hline$* *$ Idade & 0,442 & 0,176 & 0,653 & 0,393 & 0,516 & 0,792 & 0,149 & 0,796 \\
\hline $\begin{array}{l}\text { **Estado } \\
\text { Civil }\end{array}$ & 0,828 & 0,696 & 0,159 & 0,105 & 0,804 & 0,232 & 0,838 & 0,335 \\
\hline $\begin{array}{l}{ }^{* *} \text { Cor da } \\
\text { Pele }\end{array}$ & 0,915 & 0,386 & 0,927 & 0,218 & 0,772 & 0,171 & 0,191 & 0,433 \\
\hline $\begin{array}{l}\text { **Escolari } \\
\text { dade }\end{array}$ & 0,136 & 0,235 & 0,093 & 0,100 & 0,242 & 0,264 & 0,709 & 0,389 \\
\hline $\begin{array}{l}* * \text { Classe } \\
\text { Social }\end{array}$ & 0,667 & 0,167 & 0,094 & 0,853 & 0,169 & 0,309 & 0,326 & 0,706 \\
\hline $\begin{array}{l}\text { *Faz } \\
\text { atividade } \\
\text { Física }\end{array}$ & 0,127 & 0,095 & 0,793 & 0,387 & 0,303 & 0,227 & 0,173 & 0,759 \\
\hline $\begin{array}{l}{ }^{*} \text { Faz } \\
\text { Fisioterapi } \\
\text { a }\end{array}$ & $<0,001$ & $<0,001$ & 0,005 & 0,233 & 0,015 & 0,994 & 0,819 & 0,828 \\
\hline $\begin{array}{l}{ }^{* *} \text { Uso de } \\
\text { dispositivo } \\
\text { auxiliar }\end{array}$ & $<0,001$ & 0,013 & 0,329 & 0,545 & 0,206 & 0,815 & 0,011 & 0,308 \\
\hline
\end{tabular}

No domínio vitalidade os indivíduos do sexo masculino $(61,7+25,7)$ e feminino $(43,3+27,9)$, quanto à saúde mental o gênero masculino apresentou $(68,1+25,7)$ e feminino $(50,4+26,2)$ mostrando significância de $\mathrm{p}=0,001$ para ambos os domínios (Figura 2).

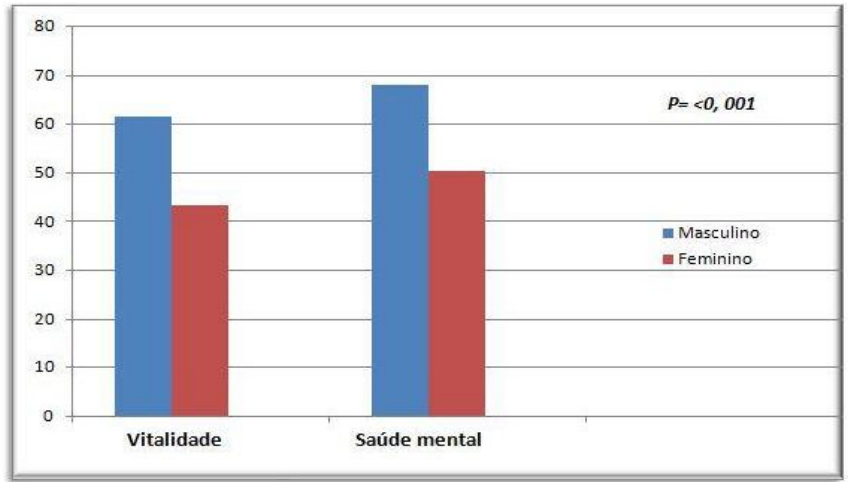


Figura 2

Associação da variável Gênero dos portadores de HTLV-I/ HAM/TSP com os domínios vitalidade e saúde mental do score SF-36, Salvador, 2010.

Em relação ao diagnóstico de HAM/TSP, a amostra constitui-se de 37 (32,2\%) indivíduos definidos, $16(13,9 \%)$ prováveis, $13(11,3 \%)$ possíveis e 49 (42,6\%) assintomáticos para HAM/TSP (Gráfico 1).

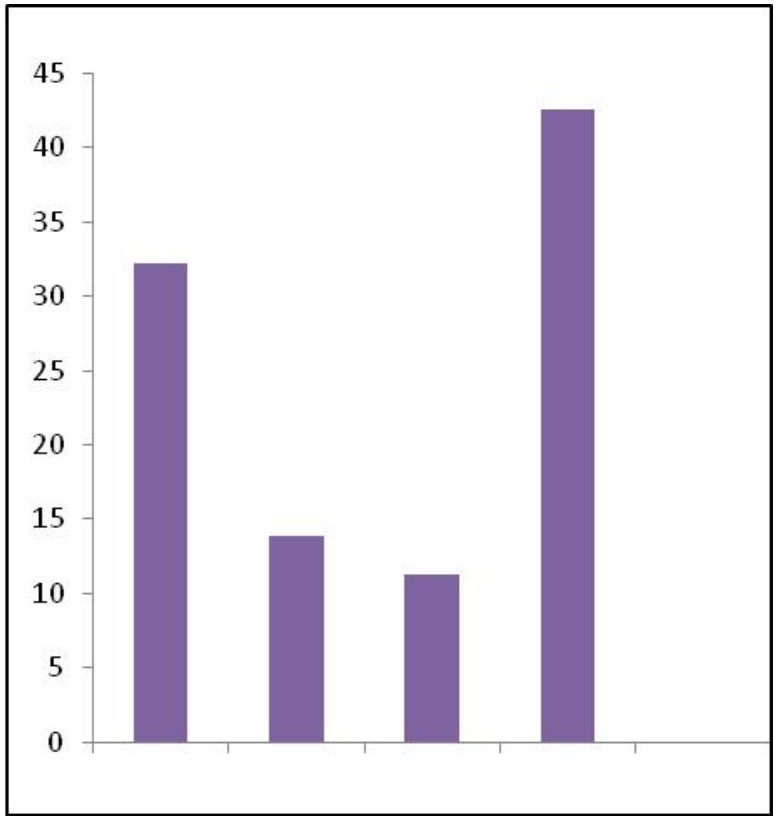

Gráfico 1

Freqüência (\%) do diagnóstico de HAM/TSP e assintomáticos para HAM/TSP na amostra de 115 portadores em Salvador-BA, 2011.

Houve associação entre alguns domínios da qualidade de vida como: capacidade funcional $(p=0,001)$, aspecto físico $(p=0,002)$ e dor $(p=0,03)$ em relação ao diagnóstico da HAM/TSP. Não houve associação quanto aos outros domínios: estado geral de saúde, vitalidade, aspectos sociais, aspectos emocionais e saúde mental.

Em relação ao domínio capacidade funcional, foi observado que 28 indivíduos (75,7\%) com diagnóstico definido para HAM/TSP apresentaram porcentagem baixa deste escore, enquanto $20(40,8 \%)$ portadores assintomáticos obtiveram escores maiores. Esta análise foi verificada através de um estudo de contingência utilizando o teste qui-quadrado (Tabela 3). 
Tabela 3

Frequência de escores (SF-36) entre o domínio capacidade funcional com o diagnóstico de HAM/TSP na amostra de 115 portadores de um centro de referência em Salvador-BA.

\begin{tabular}{|c|c|c|c|c|c|c|}
\hline \multicolumn{7}{|c|}{ Diagnóstico de HAM/TSP } \\
\hline $\begin{array}{l}\text { Capacidade } \\
\text { Funcional }\end{array}$ & & $\begin{array}{c}A^{*} \\
n \\
(\%)\end{array}$ & $\begin{array}{c}\mathrm{B}^{*} \\
\mathrm{n} \\
(\%)\end{array}$ & $\begin{array}{c}C^{*} \\
n \\
(\%)\end{array}$ & $\begin{array}{c}D^{*} \\
n \\
(\%)\end{array}$ & Total \\
\hline & $0-25 \%$ & $\begin{array}{c}28 \\
(75,7)\end{array}$ & $\begin{array}{c}9 \\
(56,2)\end{array}$ & $\begin{array}{c}7 \\
(53,8)\end{array}$ & $\begin{array}{c}9 \\
(18,4)\end{array}$ & $\begin{array}{c}53 \\
(46,1)\end{array}$ \\
\hline & $26-50 \%$ & $\begin{array}{c}5 \\
(13,5)\end{array}$ & $\begin{array}{c}5 \\
(31,2)\end{array}$ & $\begin{array}{c}3 \\
(23,1)\end{array}$ & $\begin{array}{c}12 \\
(24,5)\end{array}$ & $\begin{array}{c}25 \\
(21,7)\end{array}$ \\
\hline & $51-75 \%$ & $\begin{array}{c}2 \\
(5,4)\end{array}$ & $\begin{array}{c}1 \\
(6,2)\end{array}$ & $\begin{array}{c}3 \\
(23,1)\end{array}$ & $\begin{array}{c}8 \\
(16,3)\end{array}$ & $\begin{array}{c}14 \\
(12,2)\end{array}$ \\
\hline & $76-100 \%$ & $\begin{array}{c}2 \\
(5,4)\end{array}$ & $\begin{array}{c}1 \\
(6,2)\end{array}$ & $\begin{array}{c}0 \\
(0)\end{array}$ & $\begin{array}{c}20 \\
(40,8)\end{array}$ & $\begin{array}{c}23 \\
(20,0)\end{array}$ \\
\hline Total & & $\begin{array}{c}37 \\
(100,0)\end{array}$ & $\begin{array}{c}16 \\
(100,0)\end{array}$ & $\begin{array}{c}13 \\
(100,0)\end{array}$ & $\begin{array}{c}49 \\
(100,0)\end{array}$ & $\begin{array}{c}115 \\
(100,0)\end{array}$ \\
\hline
\end{tabular}

OBS: *A=definido, $\mathrm{B}=$ provável, $\mathrm{C}=$ possível, $\mathrm{D}=$ assintomático

Ao verificar o domínio aspecto físico verificou-se que 12 (92,3\%) indivíduos com diagnóstico possível, apresentaram baixos escores. No entanto, verificaram-se também baixos escores nos $22(44,9 \%)$ pacientes portadores do vírus, porém, assintomáticos para HAM/TSP (Tabela 4).

Tabela 4

Freqüência de escores (SF-36) entre o domínio aspecto física com o diagnóstico de HAM/TSP na amostra de 115 portadores de um centro de referência em Salvador-BA

\begin{tabular}{|c|c|c|c|c|c|c|}
\hline \multicolumn{7}{|c|}{ Diagnóstico de HAM/TSP } \\
\hline $\begin{array}{l}\text { Aspecto } \\
\text { Físico }\end{array}$ & & $\begin{array}{c}A^{*} \\
n \\
(\%)\end{array}$ & $\begin{array}{c}B^{*} \\
n \\
(\%)\end{array}$ & $\begin{array}{c}C^{*} \\
n \\
(\%)\end{array}$ & $\begin{array}{c}\mathrm{D}^{*} \\
\mathrm{n} \\
(\%)\end{array}$ & Total \\
\hline & $0-25 \%$ & $\begin{array}{c}29 \\
(78,4)\end{array}$ & $\begin{array}{c}11 \\
(68,8)\end{array}$ & $\begin{array}{c}12 \\
(92,3)\end{array}$ & $\begin{array}{c}22 \\
(44,9)\end{array}$ & $\begin{array}{c}74 \\
(64,3)\end{array}$ \\
\hline & $26-50 \%$ & $\begin{array}{c}2 \\
(5,2)\end{array}$ & $\begin{array}{c}2 \\
(12,5)\end{array}$ & $\begin{array}{c}1 \\
(7,7)\end{array}$ & $\begin{array}{c}2 \\
(4,1)\end{array}$ & $\begin{array}{c}7 \\
(6,1)\end{array}$ \\
\hline & $51-75 \%$ & $\begin{array}{c}3 \\
(8,1)\end{array}$ & $\begin{array}{c}0 \\
(0)\end{array}$ & $\begin{array}{c}0 \\
(0)\end{array}$ & $\begin{array}{c}7 \\
(14,3)\end{array}$ & $\begin{array}{c}10 \\
(8,7)\end{array}$ \\
\hline & $76-100 \%$ & $\begin{array}{c}3 \\
(8,1)\end{array}$ & $\begin{array}{c}3 \\
(18,8)\end{array}$ & $\begin{array}{c}0 \\
(0)\end{array}$ & $\begin{array}{c}18 \\
(36,7)\end{array}$ & $\begin{array}{c}24 \\
(20,9)\end{array}$ \\
\hline Total & & $\begin{array}{c}37 \\
(100,0)\end{array}$ & $\begin{array}{c}16 \\
(100,0)\end{array}$ & $\begin{array}{c}13 \\
(100,0)\end{array}$ & $\begin{array}{c}49 \\
(100,0)\end{array}$ & $\begin{array}{c}115 \\
(100,0)\end{array}$ \\
\hline
\end{tabular}

OBS: *A=definido, $\mathrm{B}=$ provável, $\mathrm{C}=$ possível, $\mathrm{D}=$ assintomático

\section{Discussão}

0 resultado desse estudo evidenciou que em relação ao perfil sociodemográfico da população estudada foi semelhante a outros estudos. Apresentou-se maior prevalência do gênero feminino, com uma proporção aproximada de 2:1 em relação ao sexo masculino, similar a outros trabalhos 5,12,13 houve um predomínio da faixa etária denominada meia 
idade (35 a 64 anos) e baixa contaminação em indivíduos adultos jovem, esse fato vem sendo abordado em estudos anteriores, mostrando que esse achado pode ser ocasionado por uma infecção passada durante o período de maior risco e que implementação de estratégias de intervenção contra a contaminação do HIV em meados de 1980 no Brasil pode ter diminuído o risco de novos casos também para o HTLV-I14 pela forma similar de contágio dessas patologias. Em relação ao estado civil houve um predomínio de pacientes casados, mas não houve uma diferença significativa entre esses e os indivíduos solteiros.

Em relação aos fatores socioeconômicos, trata-se de indivíduos menos favorecidos, com escolaridade entre primário completo e ginásio incompleto. Referente à classe social não havia nenhum indivíduo da classe A e B, mostrando maior predomínio da classe D. Outros autores, em diferentes países, têm analisado a presença de morbidade crônica segundo indicadores socioeconômicos e os achados, em geral, apontam para maior prevalência nos segmentos de menor escolaridade ou renda, além disso, em relação à economia, saúde, educação e outras desigualdades mostram-se piores, em média, nas regiões Norte e Nordeste.15

Referente ao comprometimento nas diferentes dimensões analisadas pelo questionário SF-36 em pacientes infectados pelo HTLV-I com HAM/TSP, observou-se menores valores nas dimensões aspectos físicos e capacidade funcional. Esse resultado é concordante na literatura em estudos que mostram predomínios de pontuações mais baixas obtidas pelo SF-36 referentes às dimensões limitação por aspectos físicos e capacidade funcional em paciente com HTLV-I associado a (HAM/TSP).16,17 Esses achados podem ser explicados pelo fato da (HAM/TSP) ser uma doença neurológica progressiva inflamatória do sistema nervoso central13 que provoca hipertonia bilateral nos membros inferiores, com severo encurtamento e fraqueza dos músculos pélvicos, adutores e da cadeia anterior e posterior dos membros inferiores, tornando os passos desses indivíduos arrastados e lentos, comprometendo crucialmente a marcha,18 resultando diretamente na diminuição da capacidade funcional desses pacientes.

Dentre os resultados desse estudo a variável gênero demonstrou uma correlação positiva com as variáveis relacionada à saúde mental, incluindo os domínios vitalidade, aspectos sociais, aspectos emocionais e saúde mental, mostrando que o gênero feminino possui uma maior prevalência de escores menores nesses domínios. Uma possível explicação para esse achado é o efeito psicológico que uma doença crônica pode implicar. Ser um doente crônico é lutar contra incapacidades, como no caso da paraparesia espástica decorrente do HTLV-1 que além da impossibilidade de recuperação é uma enfermidade lenta e progressiva. Estudos realizados comprovam um alto grau de ansiedade em mulheres em relação aos homens portadores de HAM/TSP, entre os principais medos é o de quem vai cuidar de seus filhos, caso atinja o maior grau de incapacidade motora, ficando extremamente restrita ao leito o que pode explicar a associação negativa entre mulheres e os domínios relacionados à saúde mental.19

Em relação à capacidade funcional evidenciou-se que indivíduos com diagnóstico definido possuem maior grau de comprometimento quando comparados aos assintomáticos para HAM/TSP. Estudos corroboram com estes achados, demonstrando o impacto negativo do aspecto funcional na qualidade de vida. Relatam que a perturbação na marcha nesses indivíduos é resultado da hipertonicidade tanto dos membros inferiores quanto dos grupos musculares pélvicos.20 Outros fatores como a redução da força muscular, fadiga e dor são responsáveis por impacto na qualidade de vida, necessitando na maioria dos casos modificarem sua independência utilizando dispositivos de assistência, como muletas, considerando que os sintomas iniciais são de fraqueza muscular em membros inferiores. 16

Quanto ao aspecto físico demonstrou que portadores com diagnóstico possível e assintomático para HAM/TSP apresentaram baixos escores. Provavelmente poderá haver associação de outras patologias, já que no diagnóstico possível não há a exclusão de outras patologias neurológicas, diferente dos outros níveis do diagnóstico. Assim como nos assintomáticos não foram afastadas outras patologias neurológicas ou do sistema 
osteomioarticular. Fazendo um parâmetro com outra patologia neurológica incapacitante e progressiva, estudos sobre a influência do aspecto físico na QV em indivíduos com esclerose múltipla verificou que há maior impacto nos domínios físicos em portadores com maior grau de comprometimento motor em relação àqueles menos comprometidos, fato também evidenciado em portadores de HAM/TSP.21

A literatura aborda que pacientes sintomáticos, apesar de terem comprometimentos importantes, podem alcançar boa independência funcional no decorrer da própria evolução da enfermidade. Estudos recentes relatam sobre a importância da fisioterapia nesses pacientes, demonstrando que programas de exercícios específicos que busquem adequação do tônus, aperfeiçoamento da flexibilidade, aumento da amplitude de movimento, fortalecimento muscular e melhora do controle postural são úteis para a recuperação funcional motora nesses indivíduos. 0 estudo demonstra que esses programas favorecem a independência funcional, minimizam complicações secundárias e dificuldades decorrentes da mobilização deficiente favorecendo a melhora da qualidade de vida nesses indivíduos. 20

\section{Conflito de Interesses}

Não houve conflito de interesses no desenvolvimento do estudo.

\section{Conclusão}

0 estudo demonstrou o perfil dos indivíduos portadores de HTLV-1 com HAM/TSP em uma amostra, sendo mais acometidos adultos, do sexo feminino e pertencente à classe D. Quanto aos domínios da qualidade de vida, portadores com diagnóstico definido para HAM/TSP apresentaram maior comprometimento em capacidade funcional e aspecto físico, mostrando que diferentes padrões de condições de vida têm impacto sobre o estado funcional e de saúde em geral. Uma limitação deste estudo foi o tipo de corte transversal, o qual não possibilita um acompanhamento desses pacientes. Sugerindo que novos estudos do tipo coorte busquem comprovações de quanto a fisioterapia é essencial para os portadores de HTLV-1 e que possam mensurar a evolução dos pacientes através da sua qualidade de vida utilizando o questionário SF-36.

\section{Referências}

1. CARNEIRO-PROIETTI A B, Ribas JGR, CATALAN-SOARES B C et al. "Infecção e Doença pelos Vírus Linfotrópicos Humanos de Células T (HTLV-I/II) no Brasil”, Revista da Sociedade Brasileira de Medicina, 35(5): 499-508. 2002.

2. Moreira Ed Jr, Ribeiro T T, Swanson P, et al. "Seroepidemiology of human T-cell Lymphotripic Virus Type I/II in northestern Brazil”, J Acquir Immune Defic Syndr, 6(8): 959-63. Aug/ 93.

3. GALVÃO-CASTRO B, Luores L, PROIETTI F, et al. "Distribution of human T-cell lymphotropic virus type I among blood donors: a nation-wide Brazilian study”, Paris: Transfusion, 37: 42. 1997.

4. Dourado I, Alcântara LCJ, Barreto ML, et al. HTLV-I in the general population of Salvador, Brazil: a city with African ethnic and sociodemographic cháracteristics. J A Acquir Immune Defic Syndr., 34(5): 527-31. Dez/ 03.

5. RIBAS J, MELO G. "Human T-cell lymphotropic virus type 1(HTLV-1)-associated myelopathy", Rev Soc Bras Med Trop, 35(4): 377-84. 2002.

6. CASKEY M. “Clinical manifestation associated with HTLV-1 infection: a cross-sectional study. AIDS” Res Hum Retroviruses, 23: 365-71. 2007.

7. CASTRO-COSTA, CM et al. "Proposal for diagnostic criteria of tropical spastic paraparesis / HTLV-1associated myelopathy (TSP/HAM); AIDS”, Res Hum Retroviruses, 22(10): 931-35. 2006.

8. MINAYO, M. C. S.; HARTZ, Z. M. A.; BUSS, P. M. “Qualidade de vida e saúde: um debate necessário”, Ciência \& Saúde Coletiva, 5: 7-18. 2000. 
9. SEIDL EMF, ZANNON CMLC. "Qualidade de vida e saúde: aspectos conceituais e metodológicos", Cad de Saúde Pública; 20: 580. 2004.

10.FLECK et al., "O instrumento de avaliação da qualidade de vida da Organização Mundial de Saúde (WHOQOL): Características e perspectivas", Red. Rev. Cient.da América Latina y el Caribe, España e Portugal, 33-38, 2000.

11.CICONELLI RM, FERRAZ MB, SANTOS W, MEINÃO I, “Quaresma MR. Tradução para a língua portuguesa e validação do questionário genérico de avaliação da qualidade de vida SF-36 (Brasil SF36)". Rev Bras Reumatol, 39: 143-50. 1999.

12. MORENO-CARVALHO OA, SANTOS JI, DI CREDICO G. "Evidence of preferential femaleprevalence of HTLV-1 associated tropical spastic paraparesis in Bahia-Brazil", Arq Neuropsiquiatr, 50: 183-88. 1992.

13.MARTINS-CASTRO LH, CHAVES CJ, CALLEGARO D, NOBREGA JP, SCAFF M. "HTLV-1 associated myelopathy in Brazil: a preliminary report", Arq Neuropsiquiatr, 7: 501-02. 1989.

14. MARQUES, MARIA CRISTINA. "Saúde e poder: a emergência política da Aids/HIV no Brasil", Hist. Cienc. Saude-Manguinhos, 9: 2002.

15. MACINTYRE S, DER G, NORRIE J. "Are there socioeconomic differences in responses to a commonly used self report measure of chronic illness?" Int J Epidemiol, 34(6): 1284-90. 2005.

16.SHUBLAQ M, ORSINI M, PUCCIONI-SOHLER M. "Implications of HAM/TSP functional incapacity in the quality of life", Arq Neuropsiquiatr. 69(2): 208-11. Apr/ 2011.

17.COUTINHO, ISA J. “Desempenho nas atividades de vida diária de indivíduos infectados pelo HTLV com TSP/HAM / Performance in activities of daily living of individuals infected with HTLV TSP / HAM. [Grassi, Maria Fernanda Rios. Salvador: s.n, 2008. 81 p. (Tese Mestrado em Medicina e Saúde Humana).

18.FRANZOI A, ARAÚJO A. "Disability profile of patients with HTLV-1 associated myelopathy/tropical spastic paraparesis using the Funcional Independence Measure-FIM", Spinal Cord, 43(4): 236-40. 2005.

19.GASCÓN, MARIA R P. Freqüência de transtorno de humor e impacto na qualidade de vida dos pacientes portadores de HTLV-1 com paraparesia espástica tropical. São Paulo, \# 121, 2010.

20.LANNES P, Neves M A O, Machado D C D, Silva J G, Bastos V H V. “Paraparesia espástica tropical: mielopatia associada ao HTLV-I: possíveis estratégias cinesioterapêuticas para melhorar padrões de marcha em portadores sintomáticos", Rev Neurocienci,14: 153-60. 2006.

21. MORALES RR, MORALES NMO, ROCHA F C G, FENELON S B, PINTO R M C, SILVA C H M. "Qualidade de vida em portadores de esclerose múltipla", Arq Neuropsiquiatr, 65: 454-60. 2007. 